Chinese Journal of Organic Chemistry

\title{
酸敏感连接单元的合成及其在两亲性嵌段共聚物中的应用
}

\author{
朱方霞 ${ }^{a}$ 庄 园 ${ }^{a, b}$ 黎 庆 ${ }^{a, b} \quad$ 杨晴来 $^{a} \quad$ 伍新燕 $^{b}$ \\ 邵志峰 $a, c$ 龚 兵*,d,e 沈玉梅*,a \\ $\left({ }^{a}\right.$ 上海交通大学系统生物医学研究院 Bio-ID 研究中心 系统生物医学教育部重点实验室 上海 200240) \\ ( ${ }^{b}$ 华东理工大学化学与分子工程学院 精细化工研究所 上海 200237) \\ (c 上海交通大学生物医学工程学院 Bio-ID 研究中心 上海 200240) \\ ( ${ }^{d}$ 北京师范大学化学学院 北京 100875) \\ (e布法罗纽约州立大学化学系 纽约 14260 美国)
}

\begin{abstract}
摘要 合成了一类基于四氢吡喃/四氢呋喃醚结构的酸敏感连接单元 $1 \mathrm{a}$ 和 $1 \mathrm{~b}$, 该连接单元的两端均为易反应的活性官 能团，其结构经 ${ }^{1} \mathrm{H} N \mathrm{NR} 、{ }^{13} \mathrm{C} N \mathrm{NR} 、 \mathrm{IR}$ 和 HRMS 表征, 并合成了一种含四氢吡喃醚结构的模型化合物以及含四氢呋 喃醚结构的两亲性嵌段共聚物, 其中聚乙二醇 $(\mathrm{PEG})$ 为亲水片段, 聚乳酸(PLA)为疏水片段, 其结构经 ${ }^{1} \mathrm{H} N \mathrm{NR} 、 \mathrm{IR}$ 以及 GPC 的表征, 并测定了该嵌段共聚物自组装胶束的粒径大小、临界胶束浓度 $(\mathrm{CMC})$ 以及常温下的稳定性. 实验结果表 明该类酸敏感嵌段共聚物自组装胶束具有良好的发展前景.
\end{abstract}

关键词 四氢吡喃醚/四氢呋喃醚酸敏感连接单元; 两亲性嵌段共聚物; 自组装胶束

\section{Synthesis and Application of pH-Sensitive Linkers in Amphiphilic Block Copolymer}

\author{
Zhu, Fangxia $^{a} \quad$ Zhuang, Yuan $^{a, b} \quad$ Li, Qing ${ }^{a, b} \quad$ Yang, Qinglai $^{a} \quad$ Wu, Xinyan ${ }^{b}$ \\ Shao, Zhifeng ${ }^{a, c}$ Gong, Bing ${ }^{*, d, e} \quad$ Shen, Yumei*,a \\ ( ${ }^{a}$ Shanghai Center for Systems Biomedicine, Key Laboratory of Systems Biomedicine and Bio-ID Center, \\ Shanghai Jiao Tong University, Shanghai 200240) \\ ( ${ }^{b}$ Key Laboratory for Advanced Materials and Institute of Fine Chemicals, East China University of \\ Science and Technology, Shanghai 200237) \\ ( ${ }^{c}$ School of Biomedical Engineering, Bio-ID Center, Shanghai Jiao Tong University, Shanghai 200240) \\ ( ${ }^{d}$ College of Chemistry, Beijing Normal University, Beijing 100875) \\ ( ${ }^{~}$ Department of Chemistry, University at Buffalo, State University of New York, Buffalo, NY 14260, USA)
}

\begin{abstract}
A series of acid cleavable linkers based on tetrahydropyran/tetrahydrofuran (THP/THF) ether were synthesized, in which both of the two terminals are active group which is easy to react with other compounds. The structures for these linkers 1a and $\mathbf{1 b}$ were confirmed by ${ }^{1} \mathrm{H}$ NMR, ${ }^{13} \mathrm{C}$ NMR, HRMS, and IR. Model compound containing THP linkage was synthesized. Furthermore a $\mathrm{pH}$-responsive amphiphilic diblock copolymer containing tetrahydrofuran (THF) linkage in which hydrophilic segment was poly(ethylene glycol) (PEG) and hydrophobic segment was hydrophobic polylactic acid (PLA) was synthesized and characterized by ${ }^{1} \mathrm{H}$ NMR, IR and gel permeation chromatography (GPC). The size, stability and critical micelle concentration (CMC) of diblock copolymer micelle were also evaluated. The preliminary experimental results show that the acid sensitive amphiphilic diblock copolymer micelle is a potential alternative for future drug delivery.
\end{abstract}

Keywords THP/THF acid cleavable linker; amphiphilic diblock copolymer, self-assembled micelle

\footnotetext{
*E-mail: yumeishen@sjtu.edu.cn; bgong@buffalo.edu

Received March 12, 2014; revised April 28, 2014; published online May 7, 2014.

Project supported by the National Natural Science Foundation of China (Nos. 81071250, 91227109), ant the Major Projects in National Science and Technology, "Creation of Major New Drugs" (No. 2011ZX09501-001-05).

国家自然科学基金(Nos. 81071250, 91227109)和新药创制重大专项(No. 2011ZX09501-001-05)资助项目.
} 
连接单元(cleavable linker)最早是在固相合成中提 出来的, 是指连接固相载体与目标化合物之间的结构片 段, 通常含双功能团并且在适当的条件下能够特异性断 裂. 在药物传递体系 ${ }^{[1]}$ 、蛋白组学 ${ }^{[2]} 、$ 影像学 ${ }^{[3,4]} 、$ DNA 测序 ${ }^{[5]}$ 等化学生物学领域有重要的应用前景.

很多药物存在水溶性差、在体内分布无选择性以及 给药后容易失活等问题, 为了解决这些问题通常选择药 物载体进行包裹 ${ }^{[6 ~ 10]}$. 目前的研究热点为智能载药体 系, 特别是酸敏感载药体系引起了广泛的关注. 研究表 明, 在正常和病理生理条件下 $\mathrm{pH}$ 值存在梯度变化 ${ }^{[11,12]}$. 肿瘤和炎症组织比血液和正常组织偏酸性. 此外, 尽管 细胞的内吞作用在接近生理 $\mathrm{pH} 7.4$ 开始, 但是在核内体 中 $\mathrm{pH}$ 为 $5.5 \sim 6.0$ 而在溶酶体中 $\mathrm{pH}$ 降低到 $4.5 \sim 5.0$ 时 内吞作用才开始. 因此酸敏感药物运输载体有望实现抗 癌药物的靶向输送, 它们在温和的酸性环境中, 酸敏感 结构破坏, 引发载药体系降解从而释放出包裹的药物. 目前研究得较多的酸敏感结构主要有原甲酸酯类、缩醛 类、缩酮类、腙类、亚胺类、三苯甲基类、肜等 ${ }^{[13 \sim 17]}$, 然 而关于四氢吡喃醚(THP)和四氢呋喃醚(THF)结构用于 酸敏感嵌段共聚物载药体系的研究却很少. Takahashi 等 ${ }^{[18]}$ 合成低聚糖时, 利用 THP 作为保护基, 在温和的酸 性条件下, 去保护而得到最终产物. Thayumanavan 等 ${ }^{[19]}$ 使用 THP 保护 2-羟基乙基甲基丙烯酸酯(HEMA)的羟基 基团合成了三重敏感的嵌段共聚物, 其中 THP 仍然是 作为保护基. Frechet 等 ${ }^{[20]}$ 以 THF 为酸敏感连接单元, 合 成了 podophyllotoxin-PEO 共聚物.

本文设计合成了四氢吡喃醚、四氢呋喃醚连接单元, 并合成了基于 THP 酸敏感结构的模型化合物以及基于 THF 的嵌段共聚物, 在此基础上考察了嵌段共聚物自组 装胶束的基本性质.

\section{1 实验部分}

\section{1 试剂与仪器}

2-羟甲基-3,4-二氢吡喃、氢化钠、三乙胺溴化茮、 对甲基苯磺酸、对硝基苯甲酰氯、10\% Pd-C、2-(7-偶氮 苯并三氮唑)- $N, N, N^{\prime}, N^{\prime}$-四甲基腿六氟磷酸酯、四丁基氟 化铵石油醚、乙酸乙酯、二氯甲烷、甲醇、四氢呋喃、 $\mathrm{N}, \mathrm{N}$-二甲基甲酰胺、氯化铵、碳酸钾、氯化钠、无水硫 酸钠、醋酸钠等分别购自百灵威、阿拉丁以及中国医药 集团试剂公司.

所用的测试仪器包括 AVANCE III 400 型核磁共振 波谱仪; EQUINOX55 型傅立叶红外光谱分析仪; Q-Tof Premier 型超高效液相色谱-四极杆飞行时间质谱联用 仪; 安捷伦 1260 型 GPC 分析色谱; JEOL JEM-100CX-II 透射电子显微镜; Malvern Zetasizer Nano $\mathrm{S}$ 激光纳米粒
度仪; F97 荧光分光光度计.

\section{2 连接单元 $1 a$ 的合成与表征}

\subsubsection{2-苯甲氧基-3,4- $2 H$-吡喃(3)的合成}

在冰水浴中, $\mathrm{N}_{2}$ 保护下, 将 $0.6 \mathrm{~g}$ 氢化钠 $(15 \mathrm{mmol})$ (60\%油中)溶解在 $10 \mathrm{~mL}$ DMF 中, 将 $1.71 \mathrm{~g} \mathrm{(15} \mathrm{mmol)} \mathrm{2-}$ 差甲基-3,4-二氢吡喃(2)缓慢滴加到 DMF 中, $0{ }^{\circ} \mathrm{C}$ 下搅 拌 $1 \mathrm{~h}$, 将 $2.64 \mathrm{~g}$ 溴化芐 $(15 \mathrm{mmol})$ 滴加到反应液中, 撤 去冰水浴, 继续摚拌 $8 \mathrm{~h}$. 用 $10 \mathrm{~mL}$ 饱和氯化铵水溶液洗 涤，乙醚萃取 $(50 \mathrm{~mL} \times 2)$, 有机相分别用水、饱和食盐水 洗, 无水硫酸钠干燥, 减压旋去溶剂, 柱层析 $[V$ (石油 醚)：V(乙酸乙酯 $)=60 ： 1$ ]得产品无色油状液体 ${ }^{[21]} 2.54$ $\mathrm{g}$, 产率 83\%. ${ }^{1} \mathrm{H}$ NMR $\left(400 \mathrm{MHz}, \mathrm{CDCl}_{3}\right) \delta: 7.40 \sim 7.26$ $(\mathrm{m}, 5 \mathrm{H}), 6.43(\mathrm{~d}, J=8.4 \mathrm{~Hz}, 1 \mathrm{H}), 4.73 \sim 4.68(\mathrm{~m}, 1 \mathrm{H})$, $4.62 \sim 4.60(\mathrm{~m}, 2 \mathrm{H}), 4.08 \sim 4.01(\mathrm{~m}, 1 \mathrm{H}), 3.61(\mathrm{dd}, J=$ $13.6,8.0 \mathrm{~Hz}, 1 \mathrm{H}), 3.54(\mathrm{dd}, J=13.6,5.6 \mathrm{~Hz}, 1 \mathrm{H}), 2.18 \sim$ $2.05(\mathrm{~m}, 1 \mathrm{H}), 2.04 \sim 1.93(\mathrm{~m}, 1 \mathrm{H}), 1.90 \sim 1.83(\mathrm{~m}, 1 \mathrm{H})$, $1.78 \sim 1.64(\mathrm{~m}, 1 \mathrm{H})$.

\subsection{2 [6-(2-溴乙氧基)四氢- $2 H$-吡喃-2-基 $]$-甲醇(4)的合} 成

$0.625 \mathrm{~g} \mathrm{2}$-溴乙醇 $(5 \mathrm{mmol})$ 溶于 $5 \mathrm{~mL}$ 二氯甲烷, 加 入 $0.25 \mathrm{~g}$ 吡啶对甲苯磺酸盐 $(1 \mathrm{mmol})$, 搅拌 $5 \mathrm{~min}$ 后, 再 加入 $1.02 \mathrm{~g}$ 化合物 $3(5 \mathrm{mmol})$, 室温下摚拌 $6 \mathrm{~h}$. 减压旋 去溶剂, 然后加入适量水, 用乙酸乙酯萃取, 有机相分 别用水、饱和食盐水洗, 无水硫酸钠干燥, 柱层析 $[V$ (石 油醚)：V(乙酸乙酯 $)=60 ： 1$ ]得产品无色油状液体 1.5 $\mathrm{g}$, 产率 91\%. 核磁共振谱图显示产物为柱层析无法分 离的两种非对映异构体 ${ }^{[2]}$. Major Isomer (trans-4): ${ }^{1} \mathrm{H}$ NMR $\left(400 \mathrm{MHz}, \mathrm{CDCl}_{3}\right) \delta: 7.35 \sim 7.26(\mathrm{~m}, 5 \mathrm{H}), 4.93(\mathrm{~d}$, $J=2.3 \mathrm{~Hz}, 1 \mathrm{H}), 4.61 \sim 4.54(\mathrm{~m}, 2 \mathrm{H}), 4.05 \sim 3.96(\mathrm{~m}, 2 \mathrm{H})$, $3.87 \sim 3.76(\mathrm{~m}, 1 \mathrm{H}), 3.58 \sim 3.41(\mathrm{~m}, 4 \mathrm{H}), 1.91 \sim 1.79(\mathrm{~m}$, $1 \mathrm{H}), 1.75 \sim 1.67(\mathrm{~m}, 1 \mathrm{H}), 1.67 \sim 1.54(\mathrm{~m}, 3 \mathrm{H}), 1.51 \sim 1.37$ (m, 1H). Minor Isomer (cis-4): ${ }^{1} \mathrm{H}$ NMR $(400 \mathrm{MHz}$, $\mathrm{CDCl}_{3}$, distinctive peaks) $\delta: 4.48(\mathrm{dd}, J=9.2,2.0 \mathrm{~Hz}, 1 \mathrm{H})$, 4.14 (ddd, $J=12.8,8.8,5.6 \mathrm{~Hz}, 1 \mathrm{H}), 3.70 \sim 3.63(\mathrm{~m}, 1 \mathrm{H})$. 1.2.3 [6-(2-溴乙氧基)四氢- $2 H$-吡喃-2-基]-甲醇 (5) 的合成

$0.11 \mathrm{~g}$ 化合物 $4(0.34 \mathrm{mmol})$ 溶于 $4 \mathrm{~mL}$ 甲醇, 加入 $15 \mathrm{mg} 10 \%$ 钯/碳, 通入氢气, 室温下反应 $1 \mathrm{~h}$. 过滤, 减 压旋去溶剂, 得 $78 \mathrm{mg}$ 无色油状液体, 产率 97\%. 核磁 共振谱图显示产物为柱层析无法分离的两种非对映异 构体 ${ }^{[22]}$. Major Isomer (trans-5): ${ }^{1} \mathrm{H}$ NMR $(400 \mathrm{MHz}$, $\left.\mathrm{CD}_{3} \mathrm{OD}\right) \delta: 4.89(\mathrm{~d}, J=2.0 \mathrm{~Hz}, 1 \mathrm{H}), 4.01 \sim 3.95(\mathrm{~m}, 1 \mathrm{H})$, $3.89 \sim 3.79(\mathrm{~m}, 1 \mathrm{H}), 3.79 \sim 3.73(\mathrm{~m}, 1 \mathrm{H}), 3.60 \sim 3.45(\mathrm{~m}$, $4 \mathrm{H}), 1.93 \sim 1.82(\mathrm{~m}, 1 \mathrm{H}), 1.79 \sim 1.66(\mathrm{~m}, 1 \mathrm{H}), 1.66 \sim 1.51$ $(\mathrm{m}, 3 \mathrm{H}), 1.38 \sim 1.27(\mathrm{~m}, 1 \mathrm{H}) ;{ }^{13} \mathrm{C}$ NMR $(100 \mathrm{MHz}$, 
$\left.\mathrm{CD}_{3} \mathrm{OD}\right) \delta$ : 97.31, 69.80, 67.02, 65.16, 30.47, 29.27, 26.72, 17.24. Minor Isomer (cis-5): ${ }^{1} \mathrm{H}$ NMR $\left(400 \mathrm{MHz}, \mathrm{CD}_{3} \mathrm{OD}\right.$, distinctive peaks) $\delta: 4.51(\mathrm{dd}, J=9.6,2.0 \mathrm{~Hz}, 1 \mathrm{H}), 4.16 \sim$ $4.09(\mathrm{~m}, 1 \mathrm{H}), 3.60 \sim 3.45(\mathrm{~m}, 1 \mathrm{H}) ;{ }^{13} \mathrm{C}$ NMR $(100 \mathrm{MHz}$, $\left.\mathrm{CD}_{3} \mathrm{OD}\right) \delta: 102.38,76.87,68.54,64.76,30.73,30.20$, 26.44, 21.26; IR (KBr) v: 3427, 2943, 1719, 1640, 1451, 1361, 1276, 1198, 1125, 1084, 1033, 977, 890, 856, 787, $662,570,471 \mathrm{~cm}^{-1}$. HRMS (ESI) calcd for $\mathrm{C}_{8} \mathrm{H}_{15} \mathrm{O}_{3} \mathrm{BrNa}$ $(\mathrm{M}+\mathrm{Na})^{+}$261.0102, found 261.0093 .

1.2.4 [6-(2-氨基乙氧基)四氢- $2 H$-吡喃-2-基 $]$-甲醇 (1a)的合成

$70 \mathrm{mg}$ 化合物 5 (0.29 mmol) 溶于 $3 \mathrm{~mL}$ 氨水, 室温 下反应 3 天. 加适量乙醇, 减压旋去溶剂, 得无色油状 液体 $49 \mathrm{mg} \mathrm{1a}$, 产率 96\%. 核磁共振谱图显示产物为柱 层析无法分离的两种非对映异构体 ${ }^{[22]}$. Major Isomer (trans-1a): ${ }^{1} \mathrm{H}$ NMR (400 MHz, $\left.\mathrm{CD}_{3} \mathrm{OD}\right) \delta: 4.88$ (d, $J=2.0$ $\mathrm{Hz}, 1 \mathrm{H}), 3.96 \sim 3.90(\mathrm{~m}, 1 \mathrm{H}), 3.84 \sim 3.78(\mathrm{~m}, 1 \mathrm{H}), 3.69 \sim$ $3.64(\mathrm{~m}, 1 \mathrm{H}), 3.54 \sim 3.47(\mathrm{~m}, 2 \mathrm{H}), 3.18(\mathrm{t}, J=4.8 \mathrm{~Hz}, 2 \mathrm{H})$, $1.96 \sim 1.73(\mathrm{~m}, 2 \mathrm{H}), 1.69 \sim 1.49(\mathrm{~m}, 2 \mathrm{H}), 1.43 \sim 1.28(\mathrm{~m}$, $2 \mathrm{H}) ;{ }^{13} \mathrm{C} \mathrm{NMR}\left(100 \mathrm{MHz}, \mathrm{CD}_{3} \mathrm{OD}\right) \delta: 97.66,70.02,65.07$, 63.03, 39.46, 28.98, 26.49, 17.14. Minor Isomer (cis-1a): ${ }^{1} \mathrm{H}$ NMR (400 MHz, $\mathrm{CD}_{3} \mathrm{OD}$, distinctive peaks) $\delta: 4.51$ (dd, $J=9.2,2.0 \mathrm{~Hz}, 1 \mathrm{H}), 4.01 \sim 3.97(\mathrm{~m}, 1 \mathrm{H}), 3.62 \sim 3.60$ (m, 1H); ${ }^{13} \mathrm{C}$ NMR (100 MHz, $\left.\mathrm{CD}_{3} \mathrm{OD}\right) \delta: 102.52,77.04$, $65.08,64.59,39.89,30.55,26.08,21.17$; IR (KBr) v: 3391, 2942, 1614, 1452, 1398, 1125, 1077, $1026 \mathrm{~cm}^{-1}$. HRMS (ESI) calcd for $\mathrm{C}_{8} \mathrm{H}_{18} \mathrm{NO}_{3}(\mathrm{M}+\mathrm{H})^{+}$176.1287, found 176.1288 .

\section{3 连接单元 $1 b$ 的合成与表征}

\subsection{1 (S)-5-氧代四氢呋喃-2-着酸(7)的合成}

取 $15.00 \mathrm{~g} L$-谷氨酸 $6(102 \mathrm{mmol})$, 加入 $21 \mathrm{~mL}$ 浓盐 酸和 $42 \mathrm{~mL}$ 水的混合溶剂溶解, $0{ }^{\circ} \mathrm{C}$ 下搅拌. 用 $45 \mathrm{~mL}$ 水溶解 $10.50 \mathrm{~g}$ 亚硝酸钠 $(150 \mathrm{mmol})$, 将其缓慢滴加到 反应瓶中, 在 $0{ }^{\circ} \mathrm{C}$ 继续反应 $3 \mathrm{~h}$, 室温下 $12 \mathrm{~h}$. 然后停止 反应, 旋出溶剂, 加入 $100 \mathrm{~mL}$ 乙酸乙酯溶解, 过滤, 无 水硫酸钠干燥, 过滤, 旋除溶剂, 得粗产物 $14.28 \mathrm{~g}$, 为 淡黄色油状液体. 该化合物未经纯化直接使用 ${ }^{[23]}$.

\subsection{2 (S)-5-(差圼基)二氢呋喃)-2(3H)-酮的合成}

将 $14.28 \mathrm{~g}$ 化合物 $7(110 \mathrm{mmol})$ 置于 $250 \mathrm{~mL}$ 反应瓶 中, 抽真空通氮气置换 3 次, 加入 $100 \mathrm{~mL}$ 无水四氢呋喃 摚拌使之溶解. 将反应液冷至 $-20{ }^{\circ} \mathrm{C}$ 并摚拌 $30 \mathrm{~min}$, 再将 $13 \mathrm{~mL} 10 \mathrm{~mol} \cdot \mathrm{L}^{-1}$ 硼烷/二甲硫醚溶液缓慢滴加到 反应瓶中. 滴加完毕后, 继续摚拌 $4 \mathrm{~h}$, 加入 $75 \mathrm{~mL}$ 甲醇 淬灭反应. 分出有机相, 再经无水硫酸钠干燥、过滤, 旋 去溶剂, 得到 $12.45 \mathrm{~g}$ 淡黄色油状液体 ${ }^{[23]}$. ${ }^{1} \mathrm{H}$ NMR
$\left(\mathrm{CDCl}_{3}, 300 \mathrm{MHz}\right) \delta: 4.58 \sim 4.66(\mathrm{~m}, 1 \mathrm{H}), 3.89$ (dd, $J=$ $12.6,3.0 \mathrm{~Hz}, 1 \mathrm{H}), 3.63(\mathrm{dd}, J=12.6,4.5 \mathrm{~Hz}, 1 \mathrm{H}), 2.46 \sim$ $2.68(\mathrm{~m}, 2 \mathrm{H}), 2.11 \sim 2.29(\mathrm{~m}, 2 \mathrm{H})$.

1.3.3 (S)-5-[(叔丁基二甲基硅氧基)甲基] 二氢呋喃$2(3 H)$-酮(9)的合成

将 $12.45 \mathrm{~g}$ 化合物 $8(107 \mathrm{mmol})$ 置于 $250 \mathrm{~mL}$ 反应瓶 中, 加入 $8.90 \mathrm{~g}$ 咪唑 $(129 \mathrm{mmol})$, 然后加入 $50 \mathrm{~mL}$ 二氯 甲烷. 再用 $50 \mathrm{~mL}$ 二氯甲烷溶解 $17.85 \mathrm{~g}$ 二甲基叔丁基 氯硅烷 $(118 \mathrm{mmol})$, 将其溶液缓慢滴加到反应瓶中, 滴 加完毕后, 室温下继续摚拌 $15 \mathrm{~h}$ 后停止反应, 分别用 2 $\mathrm{mol} \cdot \mathrm{L}^{-1}$ 稀盐酸、水和饱和 $\mathrm{NaHCO}_{3}$ 溶液洗涤, 无水硫酸 钠干燥, 过滤, 旋去溶剂. 残余液在 $200 \sim 300$ 目硅胶上 柱层析 $[V$ (石油醚) $: V$ (乙酸乙酯 $)=15: 1$ ], 得到 $6.48 \mathrm{~g}$ 淡黄色液体 $\mathbf{9}^{[23]}$, 从化合物 7 到化合物 9 总收率为 $28 \%$. ${ }^{1} \mathrm{H}$ NMR $\left(\mathrm{CDCl}_{3}, 400 \mathrm{MHz}\right) \delta: 4.54 \sim 4.59(\mathrm{~m}, 1 \mathrm{H}), 3.84$ (dd, $J=11.2,3.2 \mathrm{~Hz}, 1 \mathrm{H}$ ), 3.67 (dd, $J=11.2,3.2 \mathrm{~Hz}, 1 \mathrm{H}$ ), $2.40 \sim 2.61(\mathrm{~m}, 2 \mathrm{H}), 2.14 \sim 2.26(\mathrm{~m}, 2 \mathrm{H}), 0.87(\mathrm{~s}, 9 \mathrm{H})$, $0.05(\mathrm{~d}, J=4.0 \mathrm{~Hz}, 6 \mathrm{H})$.

1.3.4 (2R, $5 S)-5$-[(叔丁基二甲基硅氧基)甲基]四氢呋 喃-2-醇和(2S,5S)-5-[(叔丁基二甲基硅氧基)甲基]四氢 呋喃-2-醇(10)的合成

取 $6.48 \mathrm{~g}$ 化合物 $9(28 \mathrm{mmol})$ 加入 $60 \mathrm{~mL}$ 二氯甲烷, $-78{ }^{\circ} \mathrm{C}$ 下搅拌 $15 \mathrm{~min}$, 将 $35 \mathrm{~mL}$ 二异丁基氢化铝 $(1$ $\mathrm{mol} \cdot \mathrm{L}^{-1}$ in hexanes, $35 \mathrm{mmol}$ ) 缓慢滴加入反应瓶. -78 ${ }^{\circ} \mathrm{C}$ 下继续搅拌 $2 \mathrm{~h}$. 待反应完毕后, 向体系中加入 100 $\mathrm{mL} 0.1 \mathrm{~mol} \cdot \mathrm{L}^{-1}$ 稀盐酸淬灭, 用二氯甲烷萃取(125 $\mathrm{mL} \times 3)$, 有机相用饱和 $\mathrm{NaHCO}_{3}$ 水溶液洗涤, 无水硫酸 钠干燥, 过滤, 旋去溶剂, 得到无色液体 $(2 R, 5 S)-5$-[ (叔 丁基二甲基硅氧基)甲基]四氢呋喃-2-醇和(2S,5S)-5-[(叔 丁基二甲基硅氧基)甲基]四氢呋喃-2-醇两种顺反异构 体 ${ }^{[23]}$, 共 $5.16 \mathrm{~g}$, 产率 78\%. ${ }^{1} \mathrm{H}$ NMR 表明该化合物以反 式异构体为主, 含很少量顺式异构体, 在硅胶板上无法 分离. ${ }^{1} \mathrm{H} \mathrm{NMR}\left(\mathrm{CDCl}_{3}, 400 \mathrm{MHz}\right) \delta: 5.38 \sim 5.56(\mathrm{~m}, 1 \mathrm{H})$, $4.25 \sim 4.29(\mathrm{~m}, 1 \mathrm{H}), 3.80$ (dd, $J=10.4,2.8 \mathrm{~Hz}, 1 \mathrm{H}), 3.57$ (dd, $J=10.8,2.8 \mathrm{~Hz}, 1 \mathrm{H}), 2.16(\mathrm{~s}, 2 \mathrm{H}), 1.92 \sim 1.98(\mathrm{~m}$, 2H), 0.92 (s, 9H), 0.11 (s, 6H).

\subsection{5 化合物 $\mathbf{1 1}$ 的合成}

取 $232 \mathrm{mg}$ 化合物 $\mathbf{1 0}(1 \mathrm{mmol})$, 加入 $15 \mathrm{~mL}$ 二氯甲 烷溶解, 再依次加入 $250 \mathrm{mg}$ 2-溴乙醇 $(2 \mathrm{mmol}$ )和 50 mg Amberlyst A-15, $50{ }^{\circ} \mathrm{C}$ 回流 $2 \mathrm{~h}$. 待反应完毕后, 滤 去 A-15, 减压蒸去溶剂, 得到淡黄色油状液体 ${ }^{[24]}$. 柱层 析分离, [以 $V$ (石油醚) $: V($ 乙酸乙酯 $)=100: 1$ ] 为淋洗 剂, 在硅胶板上两种非对映异构体可以分离, 得到反式 异构体 $85 \mathrm{mg}$ 11a, 顺式异构体 $66 \mathrm{mg}$ 11b, 两种异构体 总 $151 \mathrm{mg}$ ，总收率 $45 \%$. 
$(2 R, 5 S)$ - $\{[5$-(2-溴乙氧基)四氢呋喃-2-基]甲氧基 $\}$ 叔 丁基二甲氧基硅烷(11a): ${ }^{1} \mathrm{H} \mathrm{NMR}\left(\mathrm{CDCl}_{3}, 400 \mathrm{MHz}\right) \delta$ : $5.18(\mathrm{~d}, J=4.8 \mathrm{~Hz}, 1 \mathrm{H}), 4.16 \sim 4.19(\mathrm{~m}, 1 \mathrm{H}), 3.92 \sim 3.96$ (m, $1 \mathrm{H}), 3.73 \sim 3.76(\mathrm{~m}, 1 \mathrm{H}), 3.61(\mathrm{~d}, J=4.4 \mathrm{~Hz}, 2 \mathrm{H})$, $3.46 \sim 3.50(\mathrm{~m}, 2 \mathrm{H}), 1.89 \sim 2.08(\mathrm{~m}, 3 \mathrm{H}), 1.69 \sim 1.73(\mathrm{~m}$, $1 \mathrm{H}), 0.89$ (s, 9H), $0.06(\mathrm{~d}, J=2.0 \mathrm{~Hz}, 6 \mathrm{H}) ;{ }^{13} \mathrm{C}$ NMR $\left(\mathrm{CDCl}_{3}, 100 \mathrm{MHz}\right) \delta:$ 104.67, 79.02, 67.29, 65.41, 32.08, $31.10,25.93,25.31,18.36,-5.26,-5.31$; IR (KBr) $v$ : 2954, 2929, 2858, 1465, 1254, 1102, 839, $777 \mathrm{~cm}^{-1}$. HRMS calcd for $\mathrm{C}_{13} \mathrm{H}_{27} \mathrm{O}_{3} \mathrm{SiBrNa}[\mathrm{M}+\mathrm{Na}]^{+} 361.0811$, found 361.0835 .

(2S,5S)-\{[5-(2-溴乙氧基)四氢呋喃-2-基]甲氧基 $\}$ 叔 丁基二甲氧基硅烷(11b): ${ }^{1} \mathrm{H} \mathrm{NMR}\left(\mathrm{CDCl}_{3}, 400 \mathrm{MHz}\right) \delta$ : $5.13(\mathrm{~d}, J=4.0 \mathrm{~Hz}, 1 \mathrm{H}), 4.11 \sim 4.14(\mathrm{~m}, 1 \mathrm{H}), 3.92 \sim 3.97$ (m, $1 \mathrm{H}), 3.68 \sim 3.75(\mathrm{~m}, 2 \mathrm{H}), 3.57 \sim 3.61(\mathrm{~m}, 1 \mathrm{H}), 3.44 \sim$ $3.50(\mathrm{~m}, 2 \mathrm{H}), 1.93 \sim 2.01(\mathrm{~m}, 3 \mathrm{H}), 1.78 \sim 1.80(\mathrm{~m}, 1 \mathrm{H})$, 0.90 (s, 9H), 0.07 (s, 6H); ${ }^{13} \mathrm{C} \mathrm{NMR}\left(\mathrm{CDCl}_{3}, 100 \mathrm{MHz}\right) \delta$ : 104.36, 81.26, 67.21, 67.19, 32.82, 30.97, 26.24, 25.93, $18.36,-5.25,-5.28$; IR (KBr) v: 2928, 2858, 1465, $1254,1098,840,777 \mathrm{~cm}^{-1}$. HRMS calcd for $\mathrm{C}_{13} \mathrm{H}_{27} \mathrm{O}_{3} \mathrm{Si}-$ $\mathrm{BrNa}[\mathrm{M}+\mathrm{Na}]^{+}$361.0811, found 361.0836.

1.3.6 化合物 $\mathbf{1 2}$ 的合成

取 $220 \mathrm{mg}$ 化合物 11a (trans) $(0.64 \mathrm{mmol})$, 加入 10 $\mathrm{mL}$ 四氢呋喃, 加入 $1.3 \mathrm{~mL}$ 四丁基氟化铵溶液( 1.3 $\mathrm{mmol}, 1 \mathrm{~mol} \cdot \mathrm{L}^{-1}$ in THF). 室温摚拌反应 $60 \mathrm{~min}$. 旋去 溶剂, 残余物以 $200 \sim 300$ 目硅胶柱层析分离纯化 $[V$ (石 油醚) $: V$ (乙酸乙酯 $)=5: 1$ ], 得 $120 \mathrm{mg}$ 无色油状液体 $(2 R, 5 S)$-[5-(2-溴乙氧基)四氢呋喃-2-基]甲醇(12a). 产率 83\%. ${ }^{1} \mathrm{H}$ NMR $\left(\mathrm{CDCl}_{3}, 400 \mathrm{MHz}\right) \delta: 5.19$ (d, $J=4.8 \mathrm{~Hz}$, $1 \mathrm{H}), 4.20 \sim 4.26(\mathrm{~m}, 1 \mathrm{H}), 3.93 \sim 3.96(\mathrm{~m}, 1 \mathrm{H}), 3.69 \sim 3.76$ $(\mathrm{m}, 2 \mathrm{H}), 3.44 \sim 3.52(\mathrm{~m}, 3 \mathrm{H}), 1.95 \sim 2.06(\mathrm{~m}, 3 \mathrm{H}), 1.64 \sim$ $1.68(\mathrm{~m}, 1 \mathrm{H}) ;{ }^{13} \mathrm{C}$ NMR $\left(\mathrm{CDCl}_{3}, 100 \mathrm{MHz}\right) \delta: 104.59$, 78.62, 67.34, 64.78, 32.41, 30.94, 24.87; IR (KBr) v: 3449, 2924, 1189, 1104, $1028 \mathrm{~cm}^{-1}$. HRMS calcd for $\mathrm{C}_{7} \mathrm{H}_{13} \mathrm{BrO}_{3} \mathrm{Na}[\mathrm{M}+\mathrm{Na}]^{+}$246.9946, found 246.9929; 同 理可得顺式异构体(2S,5S)-[5-(2-溴乙氧基)四氢呋喃-2基]甲醇(12b).

\subsection{7 连接单元 $1 \mathrm{~b}$ 的合成}

取 $80 \mathrm{mg}$ 化合物 12b (cis) (0.36 mmol), 加入 $3 \mathrm{~mL}$ 氨水, 室温下摚拌 $3 \mathrm{~d}$. 旋去溶剂, 得到 $56 \mathrm{mg}$ 无色油状 液体化合物( $2 S, 5 S)$-[5-(2-溴乙氧基)四氢呋喃-2-基]甲醇 (1b), 产率 97\%. ${ }^{1} \mathrm{H} \mathrm{NMR}\left(\mathrm{CDCl}_{3}, 400 \mathrm{MHz}\right) \delta: 5.15$ (d, $J=4.4 \mathrm{~Hz}, 1 \mathrm{H}), 4.26 \sim 4.32(\mathrm{~m}, 1 \mathrm{H}), 3.99 \sim 4.02(\mathrm{~m}, 1 \mathrm{H})$, $3.74 \sim 3.81(\mathrm{~m}, 2 \mathrm{H}), 3.55(\mathrm{dd}, J=12.0,5.2 \mathrm{~Hz}, 1 \mathrm{H}), 3.49$ $(\mathrm{t}, J=6.0 \mathrm{~Hz}, 2 \mathrm{H}), 1.92 \sim 2.07(\mathrm{~m}, 4 \mathrm{H}) ;{ }^{13} \mathrm{C} \mathrm{NMR}\left(\mathrm{CDCl}_{3}\right.$,
$100 \mathrm{MHz}) \delta$ : 104.74, 81.55, 67.93, 65.61, 33.25, 30.79, 24.33; IR (KBr) v: 3448, 2930, 1197, 1058, $1028 \mathrm{~cm}^{-1}$. HRMS calcd for $\mathrm{C}_{7} \mathrm{H}_{13} \mathrm{BrO}_{3} \mathrm{Na}[\mathrm{M}+\mathrm{Na}]^{+}$246.9946, found 246.9938. 同理可得反式异构体 $(2 R, 5 S)$-[5-(2-溴乙 氧基)四氢呋喃-2-基]甲醇.

\section{4 基于 THP 的模型化合物的合成(block cooligomer)}

1.4.12 2-(芐氧甲基)-6-\{2-[2-(2-甲氧基乙氧基)-乙氧 基]乙氧基\}四氢化- $2 H$-吡喃(13)的合成

在冰水中将 $715 \mathrm{mg}(4.35 \mathrm{mmol})$ 三乙二醇单甲醚, $153 \mathrm{mg}(0.80 \mathrm{mmol})$ 对甲基苯磺酸( $\mathrm{TsOH})$ 溶于 $15 \mathrm{~mL}$ 二 氯甲烷中, 搅拌 $1 \mathrm{~h}$. 将 $466 \mathrm{mg}(2.28 \mathrm{mmol})$ 化合物 3 溶 于 $3 \mathrm{~mL}$ 二氯甲烷, 然后缓慢滴加到反应液中, 反应 $1 \mathrm{~h}$ 后撤去冰水浴, 室温下继续反应 $20 \mathrm{~h}$. 旋去溶剂, 得到 米色液体. 柱层析 $[V$ (石油醚) $: V($ 乙酸乙酯 $)=6: 1,2$ : 1]得到 $680 \mathrm{mg}$ 白色固体，产率 $80.9 \%$. ${ }^{1} \mathrm{H}$ NMR 谱图表明 产物含有两种在硅胶板上无法分离的非对映异构体. Major Isomer (trans-13a): ${ }^{1} \mathrm{H} \mathrm{NMR}\left(400 \mathrm{MHz}, \mathrm{CDCl}_{3}\right) \delta$ : $7.35 \sim 7.30(\mathrm{~m}, 5 \mathrm{H}), 4.89(\mathrm{~s}, 1 \mathrm{H}), 4.56(\mathrm{~s}, 2 \mathrm{H}), 3.99 \sim 3.92$ (m, $1 \mathrm{H}), 3.86 \sim 3.80(\mathrm{~m}, 1 \mathrm{H}), 3.71 \sim 3.40(\mathrm{~m}, 13 \mathrm{H}), 3.36$ $(\mathrm{s}, 3 \mathrm{H}), 1.95 \sim 1.81(\mathrm{~m}, 2 \mathrm{H}), 1.75 \sim 1.55(\mathrm{~m}, 4 \mathrm{H})$. Minor Isomer (cis-13b): ${ }^{1} \mathrm{H}$ NMR (400 MHz, $\mathrm{CDCl}_{3}$, distinctive peaks) $\delta: 3.95\left(\mathrm{dd}, J_{1}=5.6, J_{2}=4.0 \mathrm{~Hz}, 1 \mathrm{H}\right)$.

1.4.2 \{6-\{2-[2-(2-甲基乙氧基)乙氧基]乙氧基 $\}$-四氢 化-2 $\mathrm{H}$-吡喃-2-基 $\}$ 甲醇(14)的合成

将 $680 \mathrm{mg}$ 化合物 $\mathbf{1 3}$ 溶于 $20 \mathrm{~mL}$ 甲醇中, 加入 137 $\mathrm{mg} 10 \%$ 钯/碳, 通入氢气, 反应液在 $40{ }^{\circ} \mathrm{C}$ 下摚拌 $6 \mathrm{~h}$ 后 过滤，旋去溶剂得到 $423 \mathrm{mg}$ 淡黄色液体，产率 $77 \% .{ }^{1} \mathrm{H}$ NMR 谱图表明产物含有两种在硅胶板上无法分离的非 对映异构体. Major Isomer (trans-14a): ${ }^{1} \mathrm{H}$ NMR (400 $\left.\mathrm{MHz}, \mathrm{CDCl}_{3}\right) \delta: 4.88(\mathrm{~s}, 1 \mathrm{H}), 4.04 \sim 3.95(\mathrm{~m}, 1 \mathrm{H}), 3.72 \sim$ $3.50(\mathrm{~m}, 14 \mathrm{H}), 3.37(\mathrm{~s}, 3 \mathrm{H}), 1.91 \sim 1.82(\mathrm{~m}, 1 \mathrm{H}), 1.81 \sim$ $1.74(\mathrm{~m}, 1 \mathrm{H}), 1.73 \sim 1.53(\mathrm{~m}, 3 \mathrm{H}), 1.53 \sim 1.49(\mathrm{~m}, 1 \mathrm{H})$. Minor Isomer (cis-14b): ${ }^{1} \mathrm{H}$ NMR $\left(400 \mathrm{MHz}, \mathrm{CDCl}_{3}\right.$, distinctive peaks) $\delta: 4.49(\mathrm{dd}, J=1.6,1.6 \mathrm{~Hz}, 1 \mathrm{H})$.

1.4.3 \{6-\{2-[2-(2-甲基乙氧基)乙氧基]乙氧基 $\}$-四氢 化- $2 \mathrm{H}$-吡喃-2-基\} 甲基-4-硝基苯甲酸酯(15)的合成

将 $278 \mathrm{mg}(0.62 \mathrm{mmol})$ 化合物 $\mathbf{1 4}$ 溶于 $15 \mathrm{~mL}$ 二氯 甲烷中. 在冰水中, 加入 $0.1 \mathrm{~mL}$ 三乙胺, 在 $0{ }^{\circ} \mathrm{C}$ 下搅拌 $20 \mathrm{~min}$. 加入 $288 \mathrm{mg}(1.55 \mathrm{mmol})$ 对硝基苯甲酰氯, 反应 $1 \mathrm{~h}$ 后撤去冰浴, 继续在常温下搅拌 $8 \mathrm{~h}$, 反应液用饱和 碳酸氢钠溶液洗, 二氯甲烷萃取, 有机相依次用水、饱 和食盐水洗、无水硫酸钠干燥, 旋去溶剂. 柱层析分离 $[V$ (石油醚) $: V($ 乙酸乙酯 $)=1 ： 1$ ] 得到 $200 \mathrm{mg}$ 淡黄色固 体, 产率 75\%. ${ }^{1} \mathrm{H}$ NMR 谱图表明产物含有两种在硅胶 板上无法分离的非对映异构体. Major Isomer (trans- 
15a): ${ }^{1} \mathrm{H}$ NMR (400 MHz, $\left.\mathrm{CDCl}_{3}\right) \delta: 8.30 \sim 8.19(\mathrm{~m}, 4 \mathrm{H})$, $4.89(\mathrm{~s}, 1 \mathrm{H}), 4.54 \sim 4.48(\mathrm{~m}, 1 \mathrm{H}), 4.37 \sim 4.28(\mathrm{~m}, 1 \mathrm{H})$, $4.18 \sim 4.10(\mathrm{~m}, 1 \mathrm{H}), 3.87 \sim 3.50(\mathrm{~m}, 12 \mathrm{H}), 3.35(\mathrm{~s}, 3 \mathrm{H})$, $1.99 \sim 1.84(\mathrm{~m}, 1 \mathrm{H}), 1.81 \sim 1.70(\mathrm{~m}, 1 \mathrm{H}), 1.70 \sim 1.50(\mathrm{~m}$, $3 \mathrm{H}), \quad 1.50 \sim 1.40(\mathrm{~m}, 1 \mathrm{H})$. Minor Isomer $\left(\right.$ cis-15-b): ${ }^{1} \mathrm{H}$ NMR (400 MHz, $\mathrm{CDCl}_{3}$, distinctive peaks) $\delta: 4.50(\mathrm{dd}$, $J=2.4,2.0 \mathrm{~Hz}, 1 \mathrm{H})$.

1.4.4 \{6-\{2-[2-(2-甲基乙氧基)乙氧基]乙氧基 $\}$-四氢 化- $2 H$-吡喃-2-基\}甲基-4-氨基苯甲酸酯(16)的合成

将 $400 \mathrm{mg}$ 化合物 15 溶于 $15 \mathrm{~mL}$ 甲醇中, 加入 55 $\mathrm{mg} 10 \%$ 钯/碳催化剂, 通入氢气, 在 $35{ }^{\circ} \mathrm{C}$ 下搅拌 $6 \mathrm{~h}$, 反应液过滤, 旋去溶剂得到 $250 \mathrm{mg}$ 黄色液体, 产率 66\%. ${ }^{1} \mathrm{H}$ NMR 谱图表明产物含有两种在硅胶板上无法 分离的非对映异构体. Major Isomer (trans-16a): ${ }^{1} \mathrm{H}$ NMR (400 MHz, $\left.\mathrm{CDCl}_{3}\right) \delta: 7.82(\mathrm{~d}, J=8.4 \mathrm{~Hz}, 2 \mathrm{H}), 6.60$ (d, $J=8.8 \mathrm{~Hz}, 2 \mathrm{H}), 4.88(\mathrm{~s}, 1 \mathrm{H}), 4.23 \sim 4.18(\mathrm{~m}, 2 \mathrm{H})$, $4.11 \sim 4.03(\mathrm{~m}, 1 \mathrm{H}), 3.84 \sim 3.78(\mathrm{~m}, 1 \mathrm{H}), 3.71 \sim 3.49(\mathrm{~m}$, $11 \mathrm{H}), 3.35(\mathrm{~s}, 3 \mathrm{H}), 1.95 \sim 1.81(\mathrm{~m}, 1 \mathrm{H}), 1.75 \sim 1.69(\mathrm{~m}$, $1 \mathrm{H}), 1.69 \sim 1.57(\mathrm{~m}, 3 \mathrm{H}), 1.48 \sim 1.37(\mathrm{~m}, 1 \mathrm{H})$. Minor Isomer (cis-16b): ${ }^{1} \mathrm{H}$ NMR $\left(400 \mathrm{MHz}, \mathrm{CDCl}_{3}\right.$, distinctive peaks) $\delta: 4.44(\mathrm{dd}, J=2.0,1.6 \mathrm{~Hz}, 1 \mathrm{H})$.

$1.4 .5\{6$ - $\{2$-[2-(2-甲基乙氧基)乙氧基]乙氧基 $\}$-四氢 化-2H-吡喃-2-基\}甲基-4-(2-(2-(叔丁基二甲基硅氧烷 基)丙酰氧基)丙酰胺)苯甲酸酯(17)的合成

将 $121 \mathrm{mg}$ 2-(2-(叔丁基二甲基硅氧烷基)丙酰氧基) 丙酸 $(2 \mathrm{c})(0.44 \mathrm{mmol})$ 和 $330 \mathrm{mg}$ HATU $(0.87 \mathrm{mmol})$ 溶于 $2 \mathrm{~mL} N, N$-二甲基甲酰胺(DMF)中, $\mathrm{N}_{2}$ 保护下, 在冰水浴 条件下, 加入 $0.1 \mathrm{~mL} N$-甲基吗啉, 在 $0{ }^{\circ} \mathrm{C}$ 下搅拌 $1 \mathrm{~h}$. 将 $397.5 \mathrm{mg}$ 化合物 $\mathbf{1 6}$ (0.35 mmol) 溶于 $1.5 \mathrm{~mL}$ DMF 中 加入反应液中, 撤去冰浴, 在室温下搅拌 $40 \mathrm{~h}$. 反应液 中加入几滴盐酸, 二氯甲烷萃取, 有机相依次用水、饱 和食盐水洗、无水硫酸钠干燥, 旋去溶剂得到深黄色液 体 $130 \mathrm{mg}$. 薄层层析 $[V$ (石油醚) $: V($ 乙酸乙酯 $)=1$ : 1.5 ]得到 $160 \mathrm{mg}$ 浅黄色液体, 产率 $70 \%$. ${ }^{1} \mathrm{H}$ NMR 谱图表 明产物含有两种在硅胶板上无法分离的非对映异构体. Major Isomer (trans-17-a): ${ }^{1} \mathrm{H}$ NMR $\left(400 \mathrm{MHz}, \mathrm{CDCl}_{3}\right) \delta$ : $7.98 \sim 7.93(\mathrm{~m}, 2 \mathrm{H}), 7.60 \sim 7.55(\mathrm{~m}, 2 \mathrm{H}), 5.32 \sim 5.22(\mathrm{~m}$, $1 \mathrm{H}), 4.84(\mathrm{~s}, 1 \mathrm{H}), 4.48 \sim 4.36(\mathrm{~m}, 2 \mathrm{H}), 4.22 \sim 4.19(\mathrm{~m}$, $1 \mathrm{H}), 4.10 \sim 4.02(\mathrm{~m}, 1 \mathrm{H}), 3.78 \sim 3.73(\mathrm{~m}, 1 \mathrm{H}), 3.68 \sim 3.46$ $(\mathrm{m}, 11 \mathrm{H}), 3.30(\mathrm{~s}, 3 \mathrm{H}), 1.95 \sim 1.74(\mathrm{~m}, 1 \mathrm{H}), 1.73 \sim 1.67$ (m, $1 \mathrm{H}), 1.67 \sim 1.55(\mathrm{~m}, 3 \mathrm{H}), 1.52(\mathrm{~d}, J=6.8 \mathrm{~Hz}, 3 \mathrm{H})$, $1.42(\mathrm{~d}, J=6.8 \mathrm{~Hz}, 3 \mathrm{H}), 1.46 \sim 1.35(\mathrm{~m}, 1 \mathrm{H}), 0.87 \sim 0.84$ (m, 9H), $0.08 \sim 0.04(\mathrm{~m}, 6 \mathrm{H})$. Minor Isomer $\left(\right.$ cis-17b): ${ }^{1} \mathrm{H}$ NMR $\left(400 \mathrm{MHz}, \mathrm{CDCl}_{3}\right.$, distinctive peaks) $\delta: 4.47$ (dd, $J=2.0,2.0 \mathrm{~Hz}, 1 \mathrm{H})$.
1.4.6 \{6-\{2-[2-(2-甲基乙氧基)乙氧基]乙氧基 $\}$-四氢 化- $2 \mathrm{H}$-吡喃-2-基\}甲基-4-(2-(2-羟基丙酰氧基)丙酰胺) 苯甲酸酯(18)的合成

在氮气保护下, 将 $80 \mathrm{mg}$ 化合物 $\mathbf{1 7}(0.12 \mathrm{mmol})$ 溶 于 $5 \mathrm{~mL}$ 无水四氢呋喃并加入 $0.05 \mathrm{~mL} \mathrm{NEt}_{3}$, 加入 0.18 $\mathrm{mL}$ TBAF $\left(1 \mathrm{~mol} \cdot \mathrm{L}^{-1}\right.$ solution in THF, $\left.0.18 \mathrm{mmol}\right)$, 然后 在室温条件下摚拌 $1.5 \mathrm{~h}$ 后停止反应. 旋蒸除去溶剂, 薄 层层析 $[V$ (二氯甲烷 $) ： V$ (乙酸乙酯 $)=1 ： 1$ 得到 $46.6 \mathrm{mg}$ 浅黄色液体，产率 70.6\%. ${ }^{1} \mathrm{H}$ NMR 谱图表明产物含有两 种在硅胶板上无法分离的非对映异构体. Major Isomer (trans-18a): ${ }^{1} \mathrm{H}$ NMR $\left(400 \mathrm{MHz}, \mathrm{CDCl}_{3}\right) \delta: 7.97 \sim 7.90$ $(\mathrm{m}, 2 \mathrm{H}), 7.96(\mathrm{~s}, 2 \mathrm{H}), 7.66 \sim 7.56(\mathrm{~m}, 2 \mathrm{H}), 4.84(\mathrm{~s}, 1 \mathrm{H})$, $4.42 \sim 4.37(\mathrm{~m}, 1 \mathrm{H}), 4.34 \sim 4.26(\mathrm{~m}, 1 \mathrm{H}), 4.24 \sim 4.19(\mathrm{~m}$, $2 \mathrm{H}), 4.10 \sim 4.02(\mathrm{~m}, 1 \mathrm{H}), 3.80 \sim 3.73(\mathrm{~m}, 2 \mathrm{H}), 3.70 \sim 3.45$ $(\mathrm{m}, 12 \mathrm{H}), 3.30(\mathrm{~s}, 3 \mathrm{H}), 1.92 \sim 1.80(\mathrm{~m}, 2 \mathrm{H}), 1.79 \sim 1.44$ $(\mathrm{m}, 8 \mathrm{H}), 1.41 \sim 1.20(\mathrm{~m}, 2 \mathrm{H})$. Minor Isomer $\left(\right.$ cis-18b): ${ }^{1} \mathrm{H}$ NMR $\left(400 \mathrm{MHz}, \mathrm{CDCl}_{3}\right.$, distinctive peaks) $\delta: 4.45(\mathrm{dd}$, $J=2.4,2.0 \mathrm{~Hz}, 1 \mathrm{H}) ;{ }^{13} \mathrm{C}$ NMR $\left(100 \mathrm{MHz}, \mathrm{CDCl}_{3}\right) \delta$ : 173.3, 166.1, 141.9, 130.9, 125.7, 119.0, 102.5, 73.9, 71.9, 70.6, 70.5, 68.9, 67.9, 67.1, 59.0, 30.9, 27.1, 21.6, 21.1.

\section{5 基于 THF 的两亲性嵌段共聚物的合成}

\subsubsection{5-(羟甲基)二氢呋喃-2(3H)-酮(19)的合成}

将 $20 \mathrm{~mL} 88 \%$ 的甲酸溶液和 $7.7 \mathrm{~mL} 30 \%$ 的双氧水 加入反应瓶中, 升温至 $50{ }^{\circ} \mathrm{C}$, 然后将 $5.0096 \mathrm{~g}$ 4-戊烯 酸 $(50 \mathrm{mmol}$ ) 溶于 $10 \mathrm{~mL} 88 \%$ 的甲酸, 再缓慢滴加到反 应瓶中 (15 min 滴完), 继续在 $50{ }^{\circ} \mathrm{C}$ 下搅拌 $10 \mathrm{~h}$ 后, 反应 液旋干后加入 $0.35 \mathrm{~mL}$ 浓盐酸和 $17 \mathrm{~mL}$ 甲醇, 在室温下 摚拌 $4 \mathrm{~h}$, 旋除溶剂得纯品为黄色液体 $5.8 \mathrm{~g}$, 产率 $100 \%$. ${ }^{1} \mathrm{H}$ NMR $\left(\mathrm{CDCl}_{3}, 400 \mathrm{MHz}\right) \delta: 4.65 \sim 4.56(\mathrm{~m}, 1 \mathrm{H})$, $3.89 \sim 3.81(\mathrm{~m}, 1 \mathrm{H}), 3.65 \sim 3.57(\mathrm{~m}, 1 \mathrm{H}), 2.65 \sim 2.45(\mathrm{~m}$, $2 \mathrm{H}), 2.30 \sim 2.18(\mathrm{~m}, 1 \mathrm{H}), 2.18 \sim 2.06(\mathrm{~m}, 1 \mathrm{H})$.

1.5.2 (5-氧代四氢呋喃-2-基)甲基-4-对甲苯磺酸酯 (20)的合成

将 $6.6 \mathrm{~g}$ 化合物 $19(56.9 \mathrm{mmol})$ 溶于 $80 \mathrm{~mL}$ 二氯甲烷 中, 加入 $10 \mathrm{~mL}$ 三乙胺、 $560 \mathrm{mg}$ DMAP $(4.6 \mathrm{mmol}$ )和 $10.86 \mathrm{~g} \mathrm{TsCl}(56.9 \mathrm{mmol})$, 常温下搅拌 $4 \mathrm{~h}$, 有机相用 水、饱和食盐水洗、无水硫酸钠干燥, 旋去溶剂得到棕 黄色固体. 柱层析分离 $[V$ (石油醚) $: V($ 二氯甲烷 $)=2$ : 1, 1：2], 得到纯产物 $13.5 \mathrm{~g}$, 产率 $87.7 \%$. ${ }^{1} \mathrm{H}$ NMR $\left(\mathrm{CDCl}_{3}, 400 \mathrm{MHz}\right) \delta: 7.78(\mathrm{~d}, J=8.4 \mathrm{~Hz}, 2 \mathrm{H}), 7.36(\mathrm{~d}, J=$ $8.4 \mathrm{~Hz}, 2 \mathrm{H}), 4.72 \sim 4.65(\mathrm{~m}, 1 \mathrm{H}), 4.21 \sim 4.11(\mathrm{~m}, 2 \mathrm{H})$, $2.64 \sim 2.48(\mathrm{~m}, 2 \mathrm{H}), 2.45(\mathrm{~s}, 3 \mathrm{H}), 2.40 \sim 2.30(\mathrm{~m}, 1 \mathrm{H})$, $2.17 \sim 2.07(\mathrm{~m}, 1 \mathrm{H}) ;{ }^{13} \mathrm{C}$ NMR $\left(100 \mathrm{MHz}, \mathrm{CDCl}_{3}\right) \delta$ : $176.1,145.5,132.3,130.2,128.1,76.5,70.1,28.0,23.6$, 21.8; IR (KBr) v: 3437, 2924, 1779, 1598, 1495, 1458, 
$1358,1266,1190,1176,1095,1003,957,860,812,740$, $665,569,554 \mathrm{~cm}^{-1}$.

1.5.35-[(4-硝基苯氧基)甲基] 二氢呋喃- $2(3 H)$-酮 (21) 的合成

将 $9.057 \mathrm{~g}$ 化合物 $20(33.5 \mathrm{mmol})$ 和 $5.09 \mathrm{~g} \mathrm{~K}_{2} \mathrm{CO}_{3}$

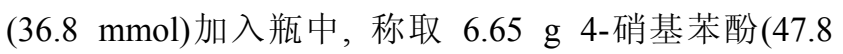
$\mathrm{mmol}$ )溶于 $60 \mathrm{~mL} \mathrm{DMF}$ 后再加入反应瓶中, $\mathrm{N}_{2}$ 保护下, $80{ }^{\circ} \mathrm{C}$ 反应 $5 \mathrm{~h}$, 二氯甲烷萃取, 有机相依次用水、饱和 食盐水洗两次, 无水 $\mathrm{NaSO}_{4}$ 干燥, 旋去溶剂得黄色固体, 柱色谱分离得 $7.67 \mathrm{~g}$ 纯品, 产率 $95.8 \% .{ }^{1} \mathrm{H} \mathrm{NMR}\left(\mathrm{CDCl}_{3}\right.$, $400 \mathrm{MHz}) \delta: 8.13(\mathrm{~d}, J=9.2 \mathrm{~Hz}, 2 \mathrm{H}), 6.94(\mathrm{~d}, J=9.2 \mathrm{~Hz}$, $2 \mathrm{H}), 4.94 \sim 4.86(\mathrm{~m}, 1 \mathrm{H}), 4.31 \sim 4.26(\mathrm{~m}, 1 \mathrm{H}), 4.19 \sim 4.13$ $(\mathrm{m}, 1 \mathrm{H}), 2.73 \sim 2.54(\mathrm{~m}, 2 \mathrm{H}), 2.49 \sim 2.39(\mathrm{~m}, 1 \mathrm{H}), 2.29 \sim$ $2.19(\mathrm{~m}, 1 \mathrm{H}) ;{ }^{13} \mathrm{C}$ NMR $\left(100 \mathrm{MHz}, \mathrm{CDCl}_{3}\right) \delta: 176.9$, 163.1, 142.0, 125.9, 114.6, 69.9, 28.3, 23.8; IR (KBr) v: 3446, 2924, 1771, 1647, 1607, 1593, 1557, 1542, 1509, 1497, 1456, 1384, 1340, 1298, 1261, 1160, 1111, 1073, 983, 944, 919, 846, 751, 689, $668 \mathrm{~cm}^{-1}$.

1.5.45-[(4-硝基苯氧基) 甲基] 二氢呋喃- $2(3 H)$-醇 (22) 的合成

称取 $6 \mathrm{~g}$ 化合物 $21(25.3 \mathrm{mmol})$ 加入反应瓶中, $\mathrm{N}_{2}$ 保护下, 加入 $40 \mathrm{~mL}$ 二氯甲烷, 冷却至 $-78{ }^{\circ} \mathrm{C}$, 缓慢 滴加 $20 \mathrm{~mL}$ 二异丁基氢化铝(DIBAL-H) $\left(1.5 \mathrm{~mol} \cdot \mathrm{L}^{-1}\right.$ in Toluene, $30 \mathrm{mmol}$ ), 反应 $4 \mathrm{~h}$ 后. 有机相依次用水、饱和 食盐水洗、无水硫酸钠干燥, 旋去溶剂得到棕黄色固体 $4.1 \mathrm{~g}$, 产率为 $67 \% .{ }^{1} \mathrm{H} \mathrm{NMR}\left(\mathrm{CDCl}_{3}, 400 \mathrm{MHz}\right) \delta: 8.21 \sim$ $8.16(\mathrm{~m}, 2 \mathrm{H}), 7.01 \sim 6.95(\mathrm{~m}, 2 \mathrm{H}), 5.65 \sim 5.55(\mathrm{~m}, 1 \mathrm{H})$, $4.67 \sim 4.60(\mathrm{~m}, 1 \mathrm{H}), 4.16 \sim 4.13(\mathrm{~m}, 1 \mathrm{H}), 4.10 \sim 4.00(\mathrm{~m}$, $1 \mathrm{H}), 2.32 \sim 2.03(\mathrm{~m}, 2 \mathrm{H}), 2.02 \sim 1.76(\mathrm{~m}, 2 \mathrm{H}) ;{ }^{13} \mathrm{C} \mathrm{NMR}$ $\left(100 \mathrm{MHz}, \mathrm{CDCl}_{3}\right) \delta: 163.7,141.9,126.1,114.7,72.9$, 69.9, 62.9, 30.6, 28.9; IR (KBr) v: 3411, 3115, 3084, 2929, $1607,1593,1510,1453,1342,1299,1264,1174,1112$, $1070,1025,967,890,847,752,691,650,500 \mathrm{~cm}^{-1}$.

1.5.5 化合物 23 (modified PEG1900)的合成

称取 $686 \mathrm{mg}$ 化合物 $22(2.87 \mathrm{mmol}) 、 3.23 \mathrm{~g} \mathrm{PEG}_{1900}$ $(1.7 \mathrm{mmol}$ )和 $600 \mathrm{mg}$ Amberlyst A-15 溶于 $60 \mathrm{~mL}$ 甲苯中, 在 $100{ }^{\circ} \mathrm{C}$ 下回流 $10 \mathrm{~h}$ 后停止反应, 反应液硅藻土过滤 除去 A-15, 旋干后得到黄色固体. 柱色谱分离得到 $1.6 \mathrm{~g}$ 较纯品, 产率 $44 \% .{ }^{1} \mathrm{H} \mathrm{NMR}\left(\mathrm{CDCl}_{3}, 400 \mathrm{MHz}\right) \delta: 8.18 \sim$ $8.13(\mathrm{~m}, 2 \mathrm{H}), 6.99 \sim 6.92(\mathrm{~m}, 2 \mathrm{H}), 5.22 \sim 5.12(\mathrm{~m}, 1 \mathrm{H})$, $4.49 \sim 4.40(\mathrm{~m}, 1 \mathrm{H}), 4.14 \sim 3.97(\mathrm{~m}, 2 \mathrm{H}), 3.87 \sim 3.39(\mathrm{~m}$, $195 \mathrm{H}), 3.34(\mathrm{~s}, 4 \mathrm{H}), 2.22 \sim 1.70(\mathrm{~m}, 4 \mathrm{H})$; IR $(\mathrm{KBr}) v$ : 3446, 2920, 2867, 1647, 1592, 1509, 1456, 1384, 1341, $1260,1108,950,850,669 \mathrm{~cm}^{-1}$.
1.5.6 化合物 $\mathbf{2 4}$ (modified $\mathrm{PEG}_{1900)}$ ) 的合成

将 $1.8 \mathrm{~g}$ 化合物 23 和 $360 \mathrm{mg} 10 \% \mathrm{Pd} / \mathrm{C}$ 溶于 $80 \mathrm{~mL}$ 甲醇中, 在 $35{ }^{\circ} \mathrm{C}$ 常压氢化、摚拌 $12 \mathrm{~h}$ 后停止反应, 反 应液过滤，旋干得浅紫色固体 $1.64 \mathrm{~g}$, 产率 $80 \%$. ${ }^{1} \mathrm{H}$ NMR $\left(\mathrm{CDCl}_{3}, 400 \mathrm{MHz}\right) \delta: 6.88 \sim 6.70(\mathrm{~m}, 4 \mathrm{H}), 5.22 \sim$ $5.10(\mathrm{~m}, 1 \mathrm{H}), 4.45 \sim 4.36(\mathrm{~m}, 1 \mathrm{H}), 3.97 \sim 3.85(\mathrm{~m}, 2 \mathrm{H})$, $3.89 \sim 3.40(\mathrm{~m}, 403 \mathrm{H}), 3.35(\mathrm{~s}, 3 \mathrm{H}), 2.17 \sim 1.91(\mathrm{~m}, 4 \mathrm{H})$; IR (KBr) v: 3446, 2954, 2859, 1647, 1509, 1456, 1384, $1348,1246,1100,947,669 \mathrm{~cm}^{-1}$.

\subsection{7 最终产物嵌段共聚物 25 [PLA-THF-PEG $(m=$} $42, n=43]$ )的合成

将 $300 \mathrm{mg}$ 一端连接丁二酸䣲的 PLA $_{3000}(0.1 \mathrm{mmol})$ 和 $77 \mathrm{mg} \mathrm{HATU}(0.2 \mathrm{mmol})$ 溶于 $15 \mathrm{~mL}$ DMF 中, 氮气保 护下, 冰水浴中加入 $0.017 \mathrm{~mL}$ DIPEA $(0.1 \mathrm{mmol}), 0{ }^{\circ} \mathrm{C}$ 下搅拌 $0.5 \mathrm{~h}$. 将 $106 \mathrm{mg}$ 化合物 $\mathbf{2 4}(0.05 \mathrm{mmol})$ 溶于 $2 \mathrm{~mL}$ $\mathrm{DMF}$, 然后加入反应瓶中并撤去冰浴, 在常温下继续摚 拌 $24 \mathrm{~h}$ 后停止反应, 反应液依次用水、饱和食盐水洗, 无水硫酸钠干燥, 旋蒸除去溶剂得到黄色液体. 柱层析 $[V$ 二氯甲烷 $): V$ (甲醇 $)=50: 1,40: 1,20: 1$ ] 得到浅黄 色固体, 产率 $50 \% .{ }^{1} \mathrm{H} \mathrm{NMR}\left(\mathrm{CDCl}_{3}, 400 \mathrm{MHz}\right) \delta: 7.55 \sim$ $7.36(\mathrm{~m}, 2 \mathrm{H}), 6.86 \sim 6.66(\mathrm{~m}, 2 \mathrm{H}), 5.35 \sim 5.26(\mathrm{~m}, 1 \mathrm{H})$, $5.22 \sim 5.05(\mathrm{~m}, 30 \mathrm{H}), 4.45 \sim 4.38(\mathrm{~m}, 1 \mathrm{H}), 3.99 \sim 3.86(\mathrm{~m}$, $2 \mathrm{H}), 3.84 \sim 3.40(\mathrm{~m}, 168 \mathrm{H}), 3.35(\mathrm{~s}, 3 \mathrm{H}), 2.77 \sim 2.50(\mathrm{~m}$, $6 \mathrm{H}), 2.21 \sim 1.80(\mathrm{~m}, 4 \mathrm{H}), 1.61 \sim 1.40(\mathrm{~m}, 90 \mathrm{H})$; IR $(\mathrm{KBr})$ $v: 3446,2932,2870,1756,1696,1511,1455,1351,1187$, $1097,952,862 \mathrm{~cm}^{-1}$.

\section{6 嵌段共聚物分子量的测定}

采用 GPC 测定嵌段共聚物 PLA-THF-PEG $(m=42$, $n=43$ ) 的分子量; 仪器: Agilent 1260 型凝胶渗透色谱仪, GPC 柱: $7.5 \mathrm{~mm} \times 300 \mathrm{~mm} 、 10 \mu \mathrm{m}$ 的凝胶色谱柱, 溶剂: 四氢呋喃, 流速: $1.0 \mathrm{~mL} / \mathrm{min}$, 柱温: $35{ }^{\circ} \mathrm{C}$, 标样: 聚苯 乙烯.

\section{7 胶束的制备及表征 \\ 1.7.1 空白胶束的制备}

准确称取 $10 \mathrm{mg}$ 合成的嵌段共聚物, 溶于 $1 \mathrm{~mL}$ $\mathrm{DMSO}$ (也可为 DMF 或 THF) 然后缓慢滴入 $8 \mathrm{~mL}$ 搅拌的 纯净水(也可为 $\mathrm{PBS}$ 缓冲溶液或生理盐水)中 $(10 \mathrm{~min} /$ $\mathrm{mL}$ ), 摚拌 $60 \mathrm{~min}$ 后转入透析袋中, 用纯净水透析 $48 \mathrm{~h}$, $4 \mathrm{~h}$ 换水 1 次, 透析除去 DMSO, 即得自组装胶束.

\subsection{2 胶束粒径分布的测定}

共聚物胶束在水介质中的直径大小及分布采用纳 米激光粒度仪 $(\mathrm{PCS})$ 测定, 取 $1 \mathrm{~mL}$ 胶束, 置入激光粒度 仪测量粒径, 用 $0.22 \mu \mathrm{m}$ 微孔滤膜过滤. 测定温度: 25 ${ }^{\circ} \mathrm{C}$, 平衡时间为 $3 \mathrm{~min}$, 激光光源: $\mathrm{He}-\mathrm{Ne}$ 激光, 波长为 
$633 \mathrm{~nm}$. 实验样本数大于 3 , 所有粒径测试结果均采用 平均值.

\subsection{3 空白胶束的稳定性试验}

空白胶束稳定性实验采用纳米粒度激光粒度仪 (PCS)测定, 取 $2 \mathrm{~mL}$ 制备好的空白胶束于 $5 \mathrm{~mL}$ 的离心 管中，隔一段时间测定胶束粒径的变化，直至粒径发生 明显变化, 说明胶束发生改变. 测定温度: $25{ }^{\circ} \mathrm{C}$, 平衡 时间为 $3 \mathrm{~min}$, 激光光源: $\mathrm{He}-\mathrm{Ne}$ 激光, 波长为 $633 \mathrm{~nm}$.

\subsection{4 最低聚集浓度或临界胶束浓度 $\mathrm{CMC}$ 的测定}

以萠为荧光分子探针(萠溶液的荧光发射光谱有 5 个苂光峰, 分别在 $373,379,384,394,480 \mathrm{~nm}$ 附近)测定 $\mathrm{CMC}$ 值，取 10 支 $10 \mathrm{~mL}$ 的 $\mathrm{EP}$ 管，每支管中加入 $100 \mu \mathrm{L}$ $6.4 \times 10^{-3} \mathrm{mmol} \cdot \mathrm{L}^{-1}$ 的萠/丙酮溶液，待丙酮挥发干后， 每支管中分别加入不同浓度(浓度范围: $0.5,0.25 \mathrm{mg} / \mathrm{mL}$, $0.1,0.05,0.025,5 \times 10^{-3}, 1 \times 10^{-3}, 0.25 \times 10^{-3}, 0.05 \times$ $10^{-3}, 0 \times 10^{-3} \mathrm{mg} / \mathrm{mL}$ ) 的胶束溶液 $2 \mathrm{~mL}$, 室温下摚拌 12 $\mathrm{h}$ 分别测定苂光强度. 苂光扫描的激发波长为 $334 \mathrm{~nm}$, 激发狭缝设置为 $5.0 \mathrm{~nm}$, 发射狭缝设置为 $2.0 \mathrm{~nm}$, 扫描 速度为 $300 \mathrm{~nm} / \mathrm{min}$. 以胶束浓度为横坐标, 荧光强度 $I_{1} / I_{3}\left(I_{1}\right.$ 对应的荧光发射波长 $\lambda_{1}$ 为 $373 \mathrm{~nm} ; I_{3}$ 对应的荧光 发射波长 $\lambda_{3}$ 为 $384 \mathrm{~nm}$ ) 的比值为纵坐标作曲线图, 通过 荧光强度 $I_{1} / I_{3}$ 的比值递增的突跃点来确定最低聚集浓度 CMC.

\section{2 结果与讨论}

\section{1 化学合成部分}

酸敏感 THP 连接单元 $\mathbf{1 a}$ 的合成路线见图 1, 从图 1 可知，首先对(3,4-二氢- $2 H$-吡喃-2-基)甲醇(2)的差基进 行保护，2-溴乙醇在酸性条件下与之继续反应, 得 2-苯 甲氧基-6-(2-溴乙氧基)四氢- $2 H$-吡喃(4), 从 ${ }^{1} \mathrm{H}$ NMR 可 知该化合物含顺反异构体, 其中反式异构体为主要产 物, 并且这两个异构体在硅胶板上无法分离 ${ }^{[22]}$. 化合物 4 去保护基苄基之后, 在浓氨水作用下, 室温下将溴高 产率地转化为氨基, 得到预期的连接单元 [6-(2-氨基乙 氧基)四氢- $2 H$-吡喃-2-基]-甲醇(1a).

在该合成路线中，如果将化合物 4 中的溴先转换为 氨基, 或者直接用 2-氨基乙醇与化合物 3 反应, 最后再 试着去掉苠基保护基, 令人意外的是该反应很难进行, 无法得到预期产物 1a.

酸敏感连接单元 $1 \mathbf{b}$ 的合成路线见图 2 , 选择 $L$-谷氨 酸为起始原料, 经过 4 步文献报道的方法得到化合物 $\mathbf{1 0}^{[23]}$, 然后与 2-溴乙醇反应, 得化合物 11, 该化合物的 两个非对映异构体在硅胶板上可以分离从而得到纯的 以得到的这两个非对映异构体 11 实际上是两个手性化 顺反异构体, 因为 5 位的构型已经由 $L$-谷氨酸确定, 所

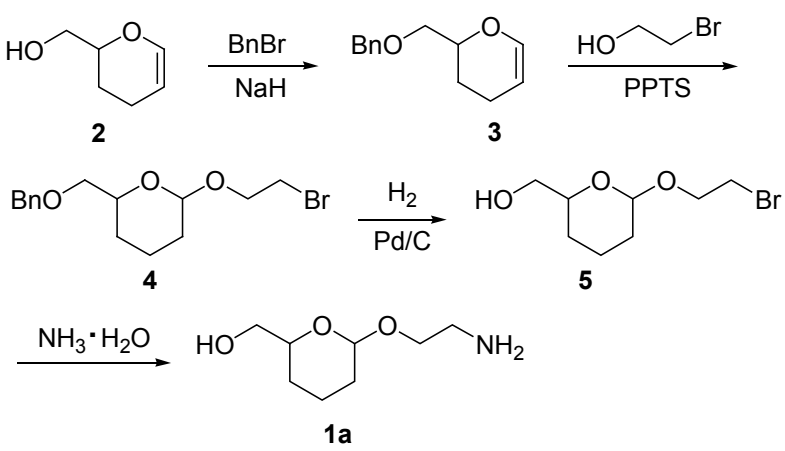

图 1 连接单元 $1 \mathbf{a}$ 的合成

Figure 1 The synthetic route of linker 1a

合物. 如果从 $D$-谷氨酸出发, 则可以得到另外两个手性 异构体，在将该连接单元用于其它化合物生物学领域, 并且需要考虑对映异构体影响时, 就可以采用这种方 法，顺利得到构型相反的对映异构体.

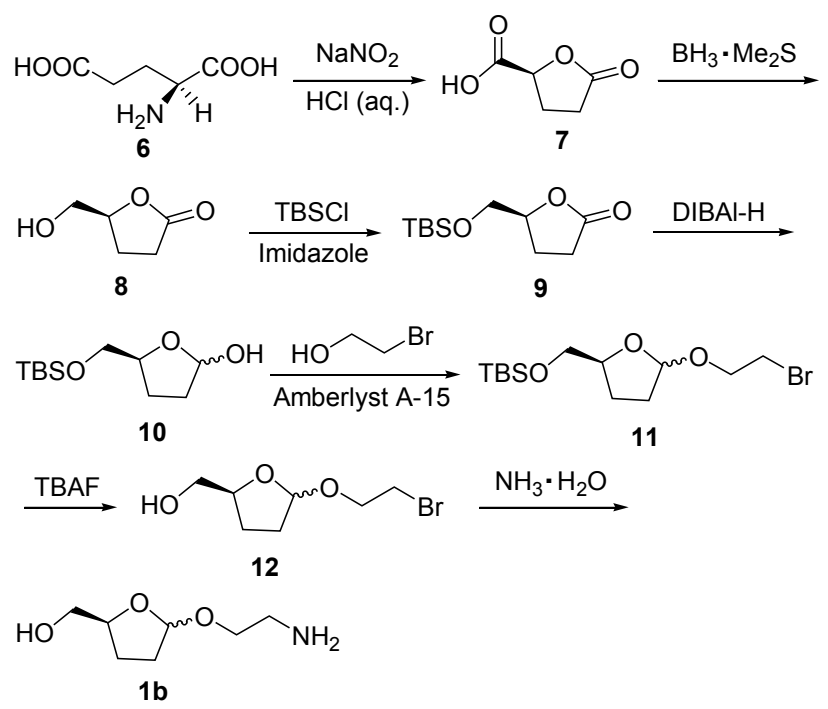

图 2 连接单元 $\mathbf{1 b}$ 的合成

Figure 2 The synthetic route of linker $\mathbf{1 b}$

拿到这样两个酸敏感连接单元，我们试着将其应 用于嵌段共聚物的合成，首先合成含 THP 结构的模型 化合物(model compound), 其合成路线见图 3, 在酸性条 件下三聚乙二醇与化合物 $\mathbf{3}$ 反应，然后去掉芐基保护基， 为了更好地跟踪监测反应的进行，引进了苯环结构，将 苯环上的硝基还原为氨基之后，与二聚乳酸反应，得小 分子的嵌段共聚物 18. 根据 ${ }^{1} \mathrm{H}$ NMR 谱图从化合物 13 开始，每一步得到的产物都是在硅胶板上无法分离的顺 反异构体混合物，并且以反式为主.

作为药物运输载体，所用的嵌段共聚物分子量不 能太小, 否则形成的胶束很不稳定, 为此我们合成了分 子量较大的基于 THF 结构的酸敏感嵌段共聚物, 其合 成路线见图 4. 为了进一步简化反应过程, 我们从 4-戊 


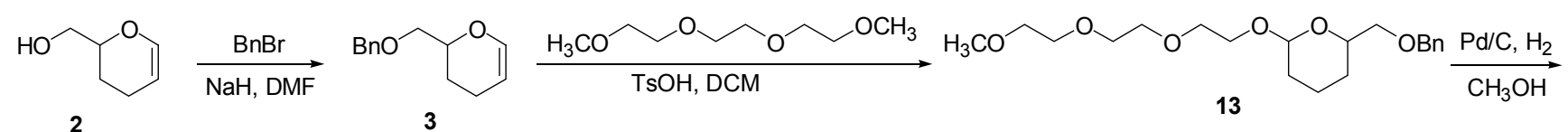<smiles>COCCOCCOCCOC1CCCC(CO)O1</smiles><smiles>COCCOCCOCCOC1CCCC(COC(=O)c2ccc([N+](=O)[O-])cc2)O1</smiles>

15

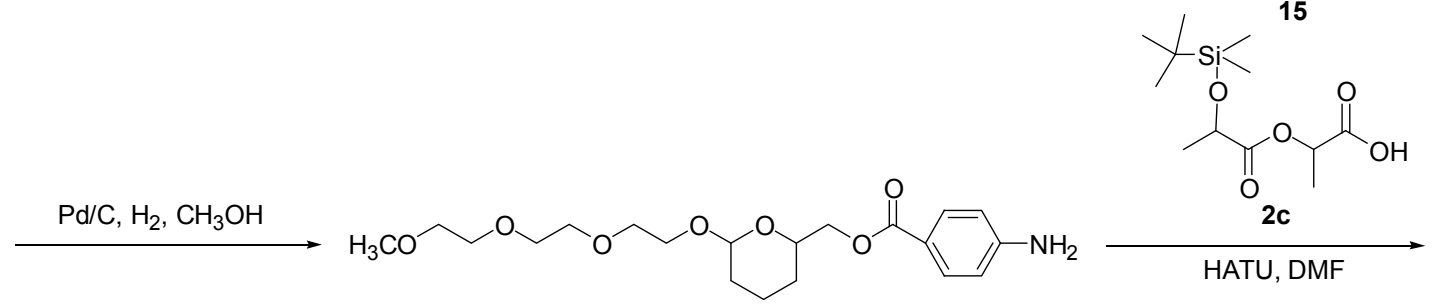

16<smiles>COCCOCCOC1CCCC(COC(=O)c2ccc(NC(=O)C(C)OC(=O)C(C)O[Si](C)(C)C(C)(C)C)cc2)O1</smiles>

17

18

图 3 模型化合物的合成

Figure 3 The synthetic route of model compound

烯酸出发, 经过七步反应得到预期的酸敏感两亲性嵌段 共聚物. 从理论上讲, 从化合物 23 开始就存在顺/反式 异构体, 但是在 ${ }^{1} \mathrm{H}$ NMR 谱图中由于高分子化合物很多 峰重叠在一起, 没有观察到顺反式异构体的特征峰.

\section{2 嵌段共聚物分子量测定及溶解性}

为了进一步确认所合成的嵌段共聚物, 对其分子量 以及分布范围进行了测定, 图 5 为凝胶渗透色谱 (GPC)
曲线, GPC 测定的平均分子量以及分子量分布(PDI)如 表 1 所示, 这些数据包括嵌段共聚物 PLA-THF-PEG $(m=42, n=43)$ 、反应原料 $\mathrm{PLA}_{3000}$ 以及中间体 24. 从图 5、表 1 可知，最终产物嵌段共聚物的分子量相比原料及 中间体的分子量明显增大且为单峰, 其分子量分布范围 PDI 较小. 该实验结果进一步表明所得到的嵌段共聚物 的合成方法是正确的，所用的分离纯化方法也比较合

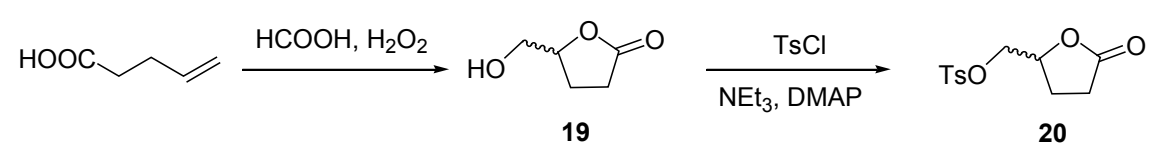<smiles>O=[N+]([O-])c1ccc(O)cc1</smiles>

20

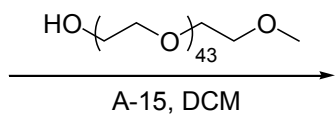<smiles>COCCCOCCOC1CCC(COc2ccc(N)cc2)O1</smiles>

23

24<smiles>CC(OC(=O)CCC(=O)O)C(=O)O[C@@H](C)C(=O)O</smiles><smiles>COCC[13CH2]CCOC1CC[C@@H](COc2ccc(NC(=O)CCC(=O)OC(C)C(=O)OC(C)C(=O)O)cc2)O1</smiles>

图 4 两亲性嵌段共聚物 PLA-THF-PEG 的合成

Figure 4 Synthesis of amphiphilic block copolymer PLA-THF-PEG 
适, 得到的产物纯度较高, 分子量分布较窄。该嵌段共 聚物在常规的有机溶剂中表现出了很好的溶解性, 例如 THF, DMF 和 DMSO 等. 另外, 两亲性嵌段共聚物由于 其本身特性可以在水溶液中自发聚集, 形成自组装胶 束.

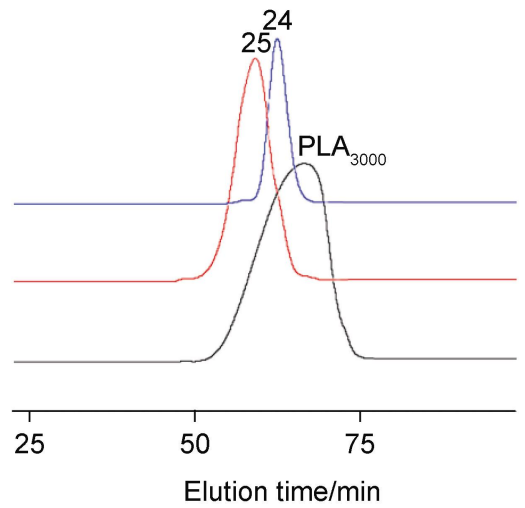

图 5 PLA-THF-PEG $(m=42, n=43)$ 的 GPC 图

Figure 5 GPC curves of PLA-THF-PEG $(m=42, n=43)$

表 1 嵌段共聚物的分子量及分子量分布范围

Table 1 Molecular weight and PDI of block copolymers

\begin{tabular}{lcccc}
\hline Polymer $^{a}$ & $M_{\mathrm{NMR}}{ }^{a}$ & $M_{\mathrm{n}}{ }^{b}$ & $M_{\mathrm{w}}{ }^{b}$ & PDI $^{b}$ \\
\hline PLA $_{3000}$ & 1000 & 1200 & 3400 & 1.28 \\
Intermediate 24 & 1900 & 2000 & 2000 & 1.03 \\
Terminal 25 & 3600 & 4300 & 4800 & 1.12 \\
\hline
\end{tabular}

${ }^{a}$ Molecular weights determined by ${ }^{1} \mathrm{H}$ NMR spectroscopy; ${ }^{b}$ Molecular weights and polydispersity determined by GPC and SM (starting material).

\section{3 胶束的制备与表征}

\subsection{1 胶束粒径分布的测定}

自组装胶束的粒径分布如图 6 所示. 从图 6 可知, 所制备的胶束是单分散的, PDI 较小, 分布较窄. 自组装 胶束的大小是一个重要的参数, $8 \mathrm{~nm}<$ 粒径 $<200 \mathrm{~nm}$ 的 胶束对肿瘤组织有一种被动靶向作用 $(\mathrm{EPR} \text { effect })^{[26]}$. 包裹抗癌药物以后，随着体内循环时间的延长而会自发 地浓集于肿瘤部位.

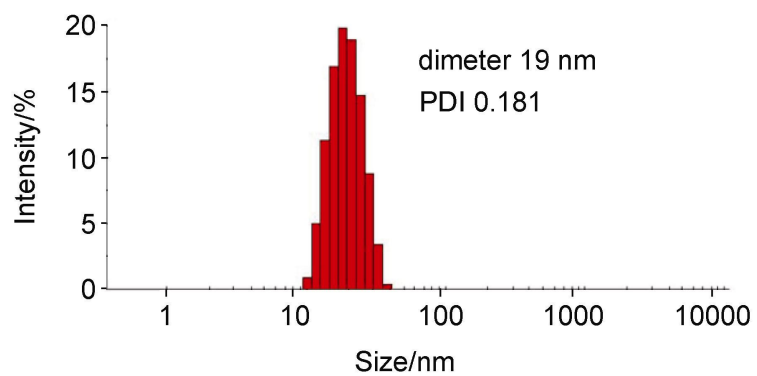

图 6 嵌段共聚物 PLA-THF-PEG $(m=42, n=43)$ 胶束的粒径 图

Figure 6 Measurement of the hydrodynamic diameter of PLA-THF-PEG $(m=42, n=43)$ micelles

\subsection{2 空白胶束的稳定性试验}

从动态光散射(DLS)实验结果(图 7)可以看出, 空白 胶束在常温下比较稳定，直到第 $10 \mathrm{~d}$ 粒径分布才由单峰 变成双峰. 说明此时胶束已经开始解体, 部分疏水片段 聚集在一起，从而观察到溶液中形成了粒径较大的胶 束. 如果继续延长反应时间，将会形成粒径更大的胶 束 $^{[27]}$.

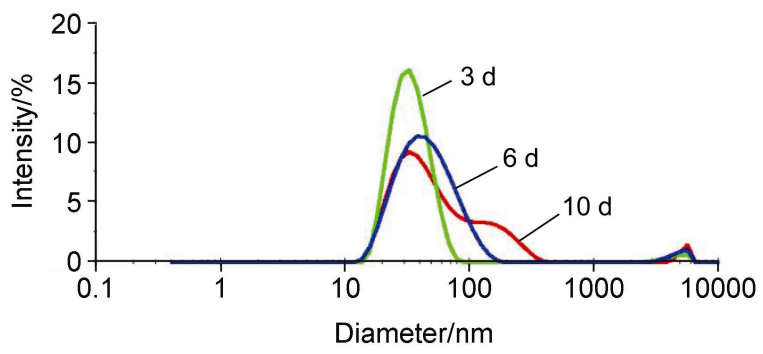

图 7 嵌段共聚物 PLA-THF-PEG $(m=42, n=43)$ 胶束的稳定 性

Figure 7 The stability of PLA-THF-PEG $(m=42, n=43)$ micelles

\subsection{3 最低聚集浓度 $\mathrm{CMC}$ 的测定}

最低聚集浓度 $(\mathrm{CMC})$ 是胶束最重要的参数之一，从 图 8 可知嵌段共聚物 PLA-THF-PEG $(m=42, n=43)$ 的 最低聚集浓度为 $0.0295 \mathrm{mg} / \mathrm{mL}$, 说明胶束在生理环境 下能够比较稳定地存在, 是一类具有发展潜力的载药胶 束. 该实验结果与粒径的稳定性实验结果一致.

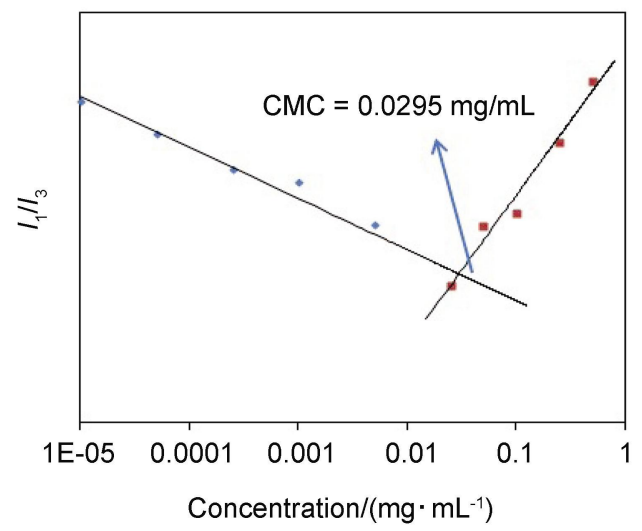

图 8 嵌段共聚物 PLA-THF-PEG $(m=42, n=43)$ 胶束的 CMC Figure 8 The CMC of PLA-THF-PEG $(m=42, n=43)$ block copolymer micelle

\section{3 结论}

合成了一类基于四氢吡喃/四氢呋喃醚( THP/THF) 结构且两端均为活性官能团的的酸敏感连接单元，并用 ${ }^{1} \mathrm{H} N M R,{ }^{13} \mathrm{C} N M R, H R M S$ 和 IR 对其进行了表征. 并合 成了基于 THP 的模型化合物(model compound)/THF 的 酸敏感两亲性嵌段共聚物(block copolymer), 其结构经 
${ }^{1} \mathrm{H}$ NMR 和 GPC 表征，并探讨了嵌段共聚物自主装胶束 的基本性质. 从实验结果可知, 所合成的酸敏感嵌段共 聚物分子量分布较窄, GPC 测定的分子量与核磁共振测 定的结果比较一致，自组装胶束大小合适、分布较窄， 在常温下比较稳定. 因此基于四氢吡喃/四氢呋喃醚结 构的嵌段共聚物自组装胶束具有较好的作为药物运输 载体的研究价值.

\section{References}

[1] Murthy, N.; Thng, Y. X.; Schuck, S. M.; Xu, C. J.; Fréchet, M. J. J. Am. Chem. Soc. 2002, 124, 12398.

[2] Barton, V.; S. Ward, A.; Chadwick, J.; Hill, A.; O'Neill, P. M. J. Med. Chem. 2010, 53, 4555.

[3] Olson, E. S.; Jiang, T.; Aguilera, T. A.; Nguyen, Q. T.; Ellies, L. G.; Scadeng, M.; Tsien, R. Y. Proc. Natl. Acad. Sci. U. S. A. 2010, 107, 4311.

[4] Louie, A. Y.; Hüber, M. M.; Ahrens, E. T.; Rothbächer, U.; Moats, R.; Jacobs, R. E.; Fraser, S. E.; Meade, T. J. Nat. Biotechnol. 2000, $18,321$.

[5] Knapp, D. C.; Serva, S.; D'Onofrio, J.; Keller, A.; Lubys, A.; Kurg, A.; Remm, M.; Engels, J. W. Chem. Eur. J. 2011, 17, 2903.

[6] Mura, S, Nicolas, J, Couvreur, P. Nat. Mater. 2013, 12, 991.

[7] Cai, X. B.; Hu, W.; Lu, W. Chin. J. Org. Chem. 2013, 33, 2520 (in Chinese). (蔡晓冰, 胡薇, 陆国林, 有机化学, 2013, 33, 2520.)

[8] Zhang, Q.; Re Ko, N.; Kwon Oh, J. Chem. Commun. 2012, 48, 7542.

[9] Xi, C. B.; Yang, D.; Li, J.; Yan, J. J.; Hu, J. H. Chin. J. Org. Chem. 2012, 32, 2166 (in Chinese). (席陈涁, 杨东, 李静, 晏建军, 胡建华, 有机化学, 2012, 32, 2166.)

[10] DiLauro, A. M.; Abbaspourrad, A.; Weitz, D. A.; Phillips, S. T. Macromolecules 2013, 46, 3309

[11] Tannock, I. F.; Rotin, D. Cancer Res. 1989, 49, 4373.

[12] Stubbs, M.; McSheehy, P. M. J.; Griffiths, J. R.; Bashford, C. L. Mol. Med. Today 2000, 6, 15.
[13] Huang, X. Macromolecules 2009, 42, 783.

[14] Zhou, L.; Yu, L.; Ding, M.; Li, J.; Tan, H.; Wang, Z.; Fu, Q. Macromolecules 2011, 44, 857.

[15] Jackson, A. W.; Stakes, C.; Fulton, D. A. Polym. Chem. 2011, 2, 2500.

[16] Prabaharan, M.; Grailer, J. J.; Pilla, S.; Steeber, D. A.; Gong, S. Biomaterials 2009, 30, 5757.

[17] Jin, Y.; Song, L.; Su, Y.; Zhu, L. J.; Pang, Y.; Qiu, F.; Tong, G. S.; Yan, D. Y.; Zhu, B. S.; Zhu, X. Y. Biomacromolecules 2011, 12, 3460.

[18] Tanaka, H.; Ishida, T.; Matoba, N.; Tsukamoto, H.; Yamada, H.; Takahashi, T. Chem. Int. Ed. 2006, 45, 6349.

[19] Klaikherd, A.; Nagamani, C.; Thayumanavan, S. J. Am. Chem. Soc. 2009, 131, 4830.

[20] Gillies, E. R.; Goodwin, A. P.; Fréchet, J. M. J. Bioconjugate Chem. 2004, 15, 1254.

[21] Carceller, E.; Merlos, M.; Giral, M.; Bartroli, J.; Garcia-Rafanell, J.; Forn, J. J. Med. Chem. 1992, 35, 676.

[22] Beaver, M. G.; Woerpel, K. A. J. Org. Chem. 2010, 75, 1107.

[23] (a) Cai, X.; Chorghade, M. S.; Fura, A.; Grewal, G. S.; Jauregui, K. A.; Lounsbury, H. A.; Scannell, R. T.; Yeh, C. G.; Young, M. A.; Yu, S. Org. Process Res. Dev. 1999, 3, 73.

(b) Pilli, R. A.; Victor, M. M.; de Meijere, A. J. Org. Chem. 2000, 65,5910 .

(c) Shiro, Y.; Kato, K.; Fujii, M.; Ida, Y.; Akita, H. Tetrahedron 2006, $62,8687$.

(d) Hamamoto, H.; Suzuki, Y.; Takahashi, H.; Ikegami, S. $A d v$. Synth. Catal. 2007, 349, 2685.

(e) Montagnat, O. D.; Lessene, G.; Hughes, A. B. J. Org. Chem. 2009, 75, 390.

[24] Dixon, D. J.; Ley, S. V.; Reynolds, D. J. Chem. Eur. J. 2002, 8, 1621.

[25] Nguyen-Ba, N.; Quimpere, M.; Kong, L. C. C.; Brown, W. L.; Dionne, G. US 5789394, 1998 [Chem. Abstr. 1998, 129, 161816].

[26] Chow, E. K.-H.; Ho, D. Sci Transl Med 2013, 5(216), 216rv4; Schmalenberg, K. E.; Frauchiger, L.; Nikkhouy-Albers, L.; Uhrich, K. E. Biomacromolecules 2001, 2, 851.

[27] Wang, D. L.; Su, Y.; Zhu, B. S.; Pang, Y.; Zhu, L. J.; Liu, J. Y.; Tu, C. L.; Yan, D. Y.; Zhu, X. Y. Biomacromolecules 2011, 12, 1370.

(Li, L.; Fan, Y.) 